\title{
Patterns of Functional Decline in Hospice: What Can Patients and Families Expect?
}

\author{
Pamela Harris, MD, FAAPMR ${ }^{1}$, Esther Wong, BA ${ }^{1}$, Sue Farrington, MBA $^{3}$, Teresa R. Craig, \\ $\mathrm{CPA}^{3}$, Joan K. Harrold, MD, Betty Oldanie, RN, BSN, MA ${ }^{5}$, Joan M. Teno, MD, MS ${ }^{4,6}$, and \\ David J. Casarett, MD, MA ${ }^{1}$ \\ ${ }^{1}$ Kansas City Hospice and Palliative Care, University of Pennsylvania Perelman School of \\ Medicine \\ ${ }^{2}$ Department of Medicine, University of Pennsylvania Perelman School of Medicine \\ ${ }^{3}$ Suncoast Solutions Incorporated, Brown University \\ ${ }^{4}$ Hospice and Community Care, Brown University \\ ${ }^{5}$ Suncoast Hospice, Brown University \\ ${ }^{6}$ Warren Alpert School of Medicine, Brown University
}

\begin{abstract}
Objectives-To describe the trajectory of patients' functional decline after they are referred to hospice.

Design-Electronic health record-based retrospective cohort study.

Setting-Three hospice programs in the southeast, northeast and Midwest US.

Participants-Hospice patients.
\end{abstract}

Main outcome measures-Palliative Performance Scale (PPS) scores measured at intervals between hospice enrollment and death, on a scale from 10-100.

Results-Of 8,669 decedents, there was an average 13.8-point decline in PPS scores. After adjusting for baseline PPS scores and length of stay in hospice, three distinct trajectories were identified, each of which was composed of two diagnoses whose rates of decline had 95\% confidence intervals that overlapped. The most rapid decline was observed for patients with cancer (adjusted decline of 8.44 points/week; 95\% CI 8.03-8.82) and stroke (7.67; 7.08-8.29). A significantly slower decline was observed in patients with pulmonary disease $(5.02 ; 4.24-5.75)$ and cardiac disease $(4.53 ; 4.05-5.05)$. Patients with debility $(1.86 ; 0.95-2.78)$ and dementia $(1.98$; 1.01-2.89) had the slowest decline. In an inverse probability-weighted sample of patients who had

Corresponding author: David Casarett MD, MA, 3615 Chestnut Street, Philadelphia, PA 19104, Phone: 610-617-2447 Fax: 610-617-2407, Casarett@mail.med.upenn.edu.

All work was performed at the University of Pennsylvania Perelman School of Medicine.

Author Contributions: All authors participated in the study's concept and design, the acquisition of data, critical revision of the manuscript, and review of the final manuscript for publication. Selected authors also participated in data analysis (David Casarett, Esther Wong) and drafting of the manuscript (Pamela Harris, David Casarett).

Conflict of Interest:

This study was funded by National Institutes of Health grant (1KM1CA156715-01) to Dr. Casarett.

The editor in chief has reviewed the conflict of interest checklist provided by the authors and has determined that the authors have no financial or any other kind of personal conflicts with this paper.

Teresa Craig and Sue Farrington are employed by Suncoast Solutions Inc., which is an industry partner of the CHOICE network. No other author has a financial relationship with Suncoast Solutions 
a PPS score recorded in the last day of life ( $n=1,959 ; 22.6 \%), 35.9 \%$ had PPS scores of at least 40, indicating some oral intake, variable mental status, limited self-care, and an ability to get out of bed for at least part of the day.

Conclusion-Although functional status generally declines among hospice patients, this decline is heterogeneous. Some patients retain some physical and cognitive function on up until the last day of life.

\section{Keywords}

Hospice; functional status; prognosis

\section{INTRODUCTION}

Patients and their families often want to know what to expect as their illness progresses. Numerous studies have shown that patients and their families want to be informed of the likely prognosis and the trajectory that an illness is likely to take. ${ }^{1-3}$ However, patients and families often don't feel that they receive as much information as they want. ${ }^{4}$

A growing body of research has begun to provide data that clinicians can use to answer these questions. For instance, studies have identified predictors of prognosis in a wide variety of settings and illnesses. ${ }^{5-7}$ Studies have also begun to define trajectories of functional decline in aging. ${ }^{8-12}$

In contrast, very little is known about the decline in function that occurs after patients enroll in hospice. ${ }^{13}$ For instance, it is not known whether all patients experience a decline in function or whether decline is slower in some patients than it is in others. Finally, it is not known what proportion of hospice patients retain some degree of functional ability in the last days of life.

Answers to these questions could help health care providers to prepare patients and families for changes that are likely to occur. Answers to these questions will also help physicians and hospices to determine Medicare hospice eligibility, which requires evidence of continued decline. Therefore, the goals of this study were to define the trajectory of functional status in a heterogeneous population of hospice patients, and to identify factors that are associated with a more rapid decline.

\section{METHODS}

\section{Setting and sample}

This study was conducted in three hospices that are pilot participants in the CHOICE network (Coalition of Hospices Organized to Investigate Comparative Effective ness). The smallest cares for 400 patients/day and the largest for 2,700. They are located in Clearwater Florida, Lancaster Pennsylvania, and Kansas City (Missouri and Kansas), and serve populations that include urban, suburban, and rural areas. All are not-for-profit.

\section{Data collection}

This study used data extracted from hospices' Electronic Health Record (EHR) systems developed by Suncoast Solutions Inc. Data were stripped of direct identifiers in order to create a HIPAA-compliant limited dataset, and transferred as an encrypted file to the University of Pennsylvania for analysis. For the main study analysis, we limited the sample to those patients who died in hospice. We reasoned that this would provide a more meaningful evaluation of functional decline in the months before death. 
As the primary measure of functional status, we extracted each patient's Palliative Performance Scale (PPS) scores at intervals during his/her hospice enrollment. The PPS is an 11-point scale of functional and cognitive status (scored from 0-100, in 10-point increments), in which a higher score reflects better function and 0 is defined as death. ${ }^{14-17}$ The PPS assesses five domains, with the following ranges: 1) Ambulation (bed-bound to full); 2) Activity (unable to work to normal); 3) Self-care (completely dependent to completely independent); 4) Intake (mouth care only to full diet); 5) Level of consciousness (drowsy or coma to fully alert). Scoring proceeds in this order, so that the first categories (e.g. ambulation, activity) are given the greatest weights. The PPS is used by all of these hospices a marker of patients' overall decline to meet Medicare eligibility criteria and thus is reliably completed.

We also extracted basic demographic variables (age, gender, race, ethnicity) and admitting diagnosis. We coded each patient's site of care at the time of enrollment (home, long term care facility, hospice inpatient unit). We also extracted clinical data elements that were likely to be markers of illness severity (e.g. oxygen, Foley catheter, presence of a pressure ulcer).

\section{Analysis}

First, we examined the distribution of PPS scores at enrollment and at the time of death, and then we calculated the net change over time. Next, because patients had multiple measurements between admission and death, we calculated the change in scores that occurred during each interval between measurements. We also calculated the average change in score/week for each interval. We excluded patients with only one PPS score, and when a patient had two PPS scores on one day we assigned an interval of 0.5 days between measurements.

To identify patient characteristics at admission that might predict a more rapid decline before death, we constructed a multivariable regression model in which the change in PPS score/week was the dependent variable. We considered all variables in Table 1 for inclusion using forward stepwise regression, and we retained only those variables that were independent predictors of decline. We also examined rates of decline by length of stay in hospice, because we reasoned that the trajectories of patients referred in the last days of life might be very different than those of patients referred much earlier. To do this, we divided the sample into 4 groups of approximately equal size (0-7 days; 8-14 days; $15-90$ days; and $>90$ days). For this and all subsequent regression models, we used robust standard errors, clustered by patient, to account for multiple PPS scores/patient.

In order to describe patients' functional status in the last days of life, we selected those patients for whom a PPS score was recorded in the last 24 hours of life. Because not all patients had a PPS score within this window, we compared patients who did vs. those who didn't in univariate logistic regression models in which documentation of a PPS score was the dependent variable. We then constructed a multivariable model using the same variable selection process described above. Using this model, we calculated the predicted likelihood that each patient in the dataset would have a completed PPS score in the last 24 hours of life, and then we used the inverse of this probability as a sampling weight in subsequent analysis to account for selective non-response. We checked the model's validity by applying the nonresponse weights to the subsample of patients for whom a PPS score was recorded. We then compared the weighted characteristics of this subgroup with those of the entire sample to confirm that weights adequately accounted for potential non-response bias. Next, we calculated the weighted distribution of PPS scores in the last day of life. We also looked for patient characteristics that predicted a better functional status at the end of life with a logistic 
regression model in which a weighted PPS score $\geq 40$ was the dependent variable, using the same modeling approach described above.

The University of Pennsylvania's Institutional Review Board approved the use of secondary data for this study. Stata statistical software (Stata MP2 11.0 for Mac, Stata Co, College Station, TX) was used for all statistical analysis.

\section{RESULTS}

Data were collected for 10,924 patients from the 3 hospices, of whom 10,334 (95\%) had more than one PPS score (Hospice \#1: $n=1,700,16.5 \%$; Hospice \#2: $n=3,644,35.3 \%$; Hospice \#3: $n=4,990,48.3 \%$ ) (See Table 1). At the time of admission, the mean PPS score was 36 and the median was 40 (range: 10-90)(Table 2). Of these, we restricted the sample to those patients $(\mathrm{n}=8,669 ; 91 \%)$ who died during a 10 -month follow-up period. Hospices documented PPS scores with varying frequencies (mean times between scores, Hospice \#1: 14.2 days; Hospice \#2: 22.2 days; Hospice \#3: 31.8 days). The final PPS scores had a mean and median of 30 (range: 10-60). On average, there was a mean 13.8-point decline. Only $321(3.9 \%)$ of these patients had an increase, while 4,767(55\%) stayed the same, and 3,528 $(40.7 \%)$ decreased. There were 32,120 pairs of measures for patients who died (Hospice \#1: 3.36 average pairs per patient; Hospice \#2: 4.04 pairs/patient; Hospice \#3: 2.37 pairs/ patient). Of these, 21,267 (66.2\%) scores didn't change between measures, while 3,950 intervals increased (12.3\%), and 6,903(21.5\%) decreased.

To describe trajectories of decline, we first examined changes in PPS score (expressed as average decline/week) as a function of length of stay. After adjusting for baseline PPS score, there was a steeper decline in function among patients with shorter lengths of stay. Compared to patients who enrolled in hospice $>90$ days before death (reference group; adjusted rate of decline in PPS score/week 1.29; 0.97-1.69), patients referred 15-90 days before death had steeper rates of decline (5.78 points/week; 95\% confidence interval 5.266.30). Rates of decline were faster among patients who enrolled in hospice $8-15$ days before death (9.49 points/week; 8.97-10.01), and fastest among those who enrolled in the last week of life (14.21 points/week; 13.70-14.72).

Next, we examined trajectories of decline among diagnostic groups, adjusting for baseline PPS scores and length of stay (in 4 categories). Three distinct groups were identified, each of which was composed of two diagnoses whose rates of decline had $95 \%$ confidence intervals that overlapped. The most rapid decline was observed for patients with cancer (adjusted decline of 8.44 points/week; 95\% CI 8.03-8.82) and stroke $(7.67 ; 7.08-8.29)$. A slower decline was observed in patients with pulmonary disease $(5.02 ; 4.24-5.75)$ and cardiac disease $(4.53 ; 4.05-5.05)$. The slowest decline was seen in patients with debility $(1.86 ; 0.95-2.78)$ and dementia $(1.98 ; 1.01-2.89)$.

Of the 8,669 patients who died, 1,959 (22.6\%) had a PPS score recorded in the last day of life (Table 2). (For patients without a PPS score in the last day, the mean elapsed time between the last measurement and death was 8 days; interquartile range: 2-12 days). Many of the patients with a measurement on the last day of life had a PPS score of 10 , which indicates drowsiness or coma, absent oral intake, and no functional ability ( $\mathrm{n}=922 ; 47.1 \%)$. However, 793 patients (40.4\%) had PPS scores of 20-30, indicating variable levels of alertness and at least some oral intake. Finally, a significant minority of patients $(n=244$; $12.5 \%$ ) had PPS scores $\geq 40$, indicating better oral intake, improved mental status, some selfcare, and an ability to get out of bed for at least part of the day.

Because patients for whom a PPS score was recorded in the last day of life may not be typical of the entire sample, we compared them with other patients who died. Patients with 
cancer were less likely to have a PPS score documented in the last day of life $(506 / 3,238$ vs. $1,453 / 5,431 ; 15.6 \%$ vs. $26.8 \%$; OR $0.51 ; 0.45-0.57 ; \mathrm{p}<0.001)$. In addition, compared to patients who died in a home setting $(500 / 3,884 ; 12.9 \%)$, patients who died in a nursing home $(469 / 2,229 ; 21 \%$; OR $1.80 ; 1.57-2.07$; $\mathrm{p}<0.001)$ were more likely to have a PPS score documented in the last day of life, as were patients in an inpatient hospice unit $(948 / 2,127$; $44.6 \%$; OR 5.44; 4.79-6.18; $\mathrm{p}<0.001)$. Finally, patients from hospice \#2 $(593 / 3,109 ; 19.1 \%$; $0.59 ; 0.51-0.68 ; \mathrm{p}<0.001)$ and $\# 3(911 / 3,967 ; 23.0 \%$; OR $0.75 ; 0.65-0.85 ; \mathrm{p}<0.001)$ were less likely than those from Hospice \#1 $(455 / 1,593 ; 28.6 \%)$ to have a PPS score documented. Using a logistic regression model derived from these variables, we calculated an inverse probability weight to account for non-random missing PPS values in the last day of life (cancer diagnosis, site of care, and hospice). Additional variables (age, race, and sex) were added until the characteristics of the patients who had PPS scores were all similar to those of the entire sample (all $p$ values $>0.25$ ). After applying these weights, the mean PPS score on the last day of life was 24.9 and the range was 10-50 (Table 2).

We used these weighted PPS scores in a multivariable model that included initial PPS score, length of stay (in 4 categories), diagnosis, initial site of care, gender, DNR status, and race. In this model, we identified several independent predictors of a PPS score $\geq 40$ in the last day of life (Table 3). For instance, among diagnoses, patients with cancer had the highest prevalence of PPS scores $\geq 40$ (25.2\%), and patients with stroke the lowest (6.1\%). Men were more likely than women to have a PPS score $\geq 40(25.7 \%$ vs. $22.1 \%)$, as were white patients $(29.6 \%$ vs. $25.1 \%)$.

\section{DISCUSSION}

The results of this study offer three main results that may help health care providers to anticipate patients' trajectory of decline in hospice. First, the net decline in PPS scores in this population was relatively small. Moreover, approximately half of patients had equal first and last PPS scores. This is not surprising, since the median length of stay in hospice is only approximately 3 weeks. ${ }^{18}$

Second, these results suggest that the decline in functional status among hospice patients is very heterogeneous. For instance, after adjusting for baseline PPS scores, we found an approximately five-fold variation in rates of decline across diagnoses, and we identified three distinct groups (cancer and stroke; cardiac and pulmonary disease; debility and dementia). This finding may help providers to describe likely future changes to patients and families.

Third, even after adjusting for length of stay, several characteristics (e.g. cancer diagnosis, hospice care at home, male gender and white race) were associated with higher PPS scores in the last day of life. It is unclear why some patients' function is much higher on the last day of life than others' is. In part, this result might be explained by a more gradual decline in function among some patients. In addition, though, it is also likely that some patients (e.g. those with cancer) may be more likely to experience sudden, immediately fatal complications such as a pulmonary embolus or hemorrhage. These patients might retain much of their functional ability until hours or even minutes before death.

This study has three limitations that should be noted. First, almost all of these patients were very near the end of life, with limited functional status at the time of hospice enrollment. Therefore, PPS scores were almost entirely in the lower half of the scale and are susceptible to floor effects that may mask a decline over time..${ }^{15,16,19}$ However, the PPS scale is widely used in hospice and palliative care populations, ${ }^{5}$ giving these results good generalizability. 
Second, the changes in functional status reported here represent average changes per unit of time. We did not distinguish among patients who experienced an early decline vs. those who underwent a decline close to death. However, the analysis of PPS scores immediately before death provides valuable insights into the groups of patients who experience minimal or no decline up until the end of life.

Third, the results reported here come from only three hospices and may not reflect the experience of the entire hospice population. It is possible that other hospices with a different patient mix may observe different results and "open access" hospice programs that allow more aggressive treatment may also produce different results. ${ }^{20}$ Finally, because hospices use a decline in functional status as evidence of continued eligibility, it is possible that different results might be observed in hospices that place more or less emphasis on documentation.

Although a decline in function is an anticipated outcome for patients in hospice care many patients have lost most or all of their capacity for meaningful interaction before they enroll. Moreover, expectations of decline that are built into hospice eligibility requirements may not adequately reflect the trajectories of hospice patients. Therefore, health care providers have an opportunity to offer guidance and support that anticipates the likely trajectory of functional decline so that patients and their families can plan accordingly.

\section{Acknowledgments}

Sponsor's Role: The study funder had no role in the collection, analysis, and interpretation of data, in the writing of the report, or in the decision to submit the article for publication. The investigators maintained independence from funders throughout all stages of research. All authors, external and internal, had full access to all of the data (including statistical reports and tables) in the study and can take responsibility for the integrity of the data and the accuracy of the data analysis.

\section{References}

1. Russell BJ, Ward AM. Deciding what information is necessary: Do patients with advanced cancer want to know all the details? Cancer Manag Res. 2011; 3:191-199. [PubMed: 21792328]

2. Degner LF, Kristjanson LJ, Bowman D, et al. Information needs and decisional preferences in women with breast cancer. JAMA. 1997; 277:1485-1492. [PubMed: 9145723]

3. Strohbuecker B, Gaertner J, Stock S. Informing severely ill patients: Needs, shortcomings and strategies for improvement. Breast Care. 2011; 6:8-13. [PubMed: 21547020]

4. Rainbird K, Perkins J, Sanson-Fisher R, et al. The needs of patients with advanced, incurable cancer. Br J Cancer. 2009; 101:759-764. [PubMed: 19654579]

5. Hanks GW, Robbins M. The imPaCT study: A randomised controlled trial to evaluate a hospital palliative care team. Br J Cancer. 2002; 87:733-739. [PubMed: 12232756]

6. Coventry PA, Grande GE, Richards DA, et al. Prediction of appropriate timing of palliative care for older adults with non-malignant life-threatening disease: A systematic review. Age Ageing. 2005; 34:218-227. [PubMed: 15863407]

7. Mitchell SL, Miller SC, Teno JM, et al. Prediction of 6-month survival of nursing home residents with advanced dementia using ADEPT vs hospice eligibility guidelines. JAMA. 2010; 304:19291935. [PubMed: 21045099]

8. Kelley AS, Ettner SL, Morrison RS, et al. Disability and decline in physical function associated with hospital use at end of life. J Gen Intern Med. 2012; 27:794-800. [PubMed: 22382455]

9. Covinsky KE, Eng C, Lui LY, et al. The last 2 years of life: Functional trajectories of frail older people. J Am Geriatr Soc. 2003; 51:492-498. [PubMed: 12657068]

10. Teno JM, Mor V, Ward N, et al. Bereaved family member perceptions of quality of end-of-life care in U.S. regions with high and low usage of intensive care unit care. J Am Geriatr Soc. 2005; 53:1905-1911. [PubMed: 16274371] 
11. Abicht-Swensen LM, Debner LK. The Minimum Data Set 2. 0: A functional assessment to predict mortality in nursing home residents. Am J Hospic Palliat Care. 1999; 16:527-532.

12. Lunney JR, Lynn J, Foley DJ, et al. Patterns of functional decline at the end of life. JAMA. 2003; 289:2387-2392. [PubMed: 12746362]

13. Downing GM, Lesperance M, Lau F, et al. Survival implications of sudden functional decline as a sentinel event using the palliative performance scale. J Palliat Med. 2010; 13:549-557. [PubMed: 20377498]

14. Younis T, Milch R, Abul-Khoudoud N, et al. Length of survival in hospice for cancer patients referred from a comprehensive cancer center. Am J Hosp Palliat Med. 2009; 26:281-287.

15. Weng L-C, Huang H-L, Wilkie DJ, et al. Predicting survival with the Palliative Performance Scale in a minority-serving hospice and palliative care program. J Pain Symptom Manage. 2009; 37:642-648. [PubMed: 18823751]

16. Harrold J, Rickerson E, McGrath J, et al. Is the Palliative Performance Scale a useful predictor of mortality in a heterogeneous hospice population? J Palliat Med. 2005; 8:503-509. [PubMed: 15992191]

17. Morita T, Tsunoda J, Inoue S, et al. Validity of the palliative performance scale from a survival perspective. J Pain Symptom Manage. 1999; 18:2-3. [PubMed: 10439564]

18. National Hospice and Palliative Care Organization Facts and Figures. Hospice Care in America. 2010. http://www.nhpco.org/files/public/Statistics_Research/ Hospice_Facts_Figures_Oct-2010.pdf

19. Head B, Ritchie CS, Smoot TM. Prognostication in hospice care: can the palliative performance scale help? J Palliat Med. 2005; 8:492-502. [PubMed: 15992190]

20. Casarett D. Rethinking hospice eligibility criteria. JAMA. 2011; 305:1031-1032. [PubMed: 21386081] 
Table 1

Characteristics of hospice decedents $(n=8,669)$.

\begin{tabular}{|c|c|}
\hline \multicolumn{2}{|l|}{ Patient characteristics } \\
\hline Age: mean & 75 \\
\hline \multicolumn{2}{|l|}{ Gender } \\
\hline Male & $3640(42.0 \%)$ \\
\hline \multicolumn{2}{|l|}{ Hospice } \\
\hline$\# 1$ & $1,456(16.8 \%)$ \\
\hline$\# 2$ & $3,051(35.2 \%)$ \\
\hline \#3 & $4,162(48.0 \%)$ \\
\hline \multicolumn{2}{|l|}{ Admitting diagnosis } \\
\hline Cancer & $2,887(33.3 \%)$ \\
\hline Debility & $1,240(14.3 \%)$ \\
\hline Cardiovascular disease & $1,283(14.8 \%)$ \\
\hline Dementia & $1,031(11.9 \%)$ \\
\hline Pulmonary disease & $607(7.0 \%)$ \\
\hline Stroke & $425(4.9 \%)$ \\
\hline Other & $1,196(13.8 \%)$ \\
\hline \multicolumn{2}{|l|}{ Initial site of care } \\
\hline Home & $3,996(46.1 \%)$ \\
\hline Nursing Home & $2,263(26.1 \%)$ \\
\hline Hospital & $1,951(22.0 \%)$ \\
\hline Other ${ }^{*}$ & $459(5.3 \%)$ \\
\hline Married & $2,505(28.9 \%)$ \\
\hline Foley catheter & $1,864(21.5 \%)$ \\
\hline Oxygen & $4,222(48.7 \%)$ \\
\hline Do Not Resuscitate order on admission & $6,614(76.3 \%)$ \\
\hline Advanced Directive & $3,398(39.2 \%)$ \\
\hline Pressure ulcer & $1,162(13.4 \%)$ \\
\hline Length of stay (days): Median (Interqu & $31(7-91)$ \\
\hline
\end{tabular}

*ther: Includes group home, prison, homeless shelter, psychiatric institution. 
Table 2

Distribution of Palliative Performance Scale scores

\begin{tabular}{|l|l|l|l|}
\hline $\begin{array}{l}\text { Palliative Performance Scale } \\
\text { score }\end{array}$ & $\begin{array}{l}\text { Distribution on admission } \\
\text { (n=10,334) }\end{array}$ & $\begin{array}{l}\text { Distribution in last 24 hours of } \\
\text { life (n=1,959) }\end{array}$ & $\begin{array}{l}\text { Weighted distribution in last 24 } \\
\text { hours of life* }\end{array}$ \\
\hline 10 & $1,189(11.5 \%)$ & $922(47.1 \%)$ & $21.6 \%$ \\
\hline 20 & $1,252(12.1 \%)$ & $448(22.9 \%)$ & $19.8 \%$ \\
\hline 30 & $2,162(20.9 \%)$ & $345(17.6 \%)$ & $22.3 \%$ \\
\hline 40 & $3,060(29.6 \%)$ & $183(9.3 \%)$ & $21.7 \%$ \\
\hline 50 & $2,104(20.4 \%)$ & $57(2.9 \%)$ & $12.2 \%$ \\
\hline 60 & $471(4.6 \%)$ & $3(0.2 \%)$ & $2 \%$ \\
\hline 70 & $80(0.8 \%)$ & $1(0.05 \%)$ & $0 \%$ \\
\hline 80 & $15(0.2 \%)$ & $0(0.0 \%)$ & $0 \%$ \\
\hline 90 & $1(0.01 \%)$ & $0(0.0 \%)$ & $0 \%$ \\
\hline
\end{tabular}

* Inverse probability weights applied to account for incomplete data collection. Weighting variables included initial PPS score, diagnosis, hospice, initial site of care, age, gender, and race (white vs. non-white). 
Table 3

Independent predictors of a final Palliative Performance Scale score $\geq 40$

\begin{tabular}{|c|c|c|c|}
\hline & & p-value & Adjusted proportion \\
\hline \multicolumn{4}{|l|}{ Diagnoses } \\
\hline Cancer & - & - & $25.2 \%$ \\
\hline Debility & $0.53(0.45-0.64)$ & $<0.001$ & $13.1 \%$ \\
\hline Dementia & $0.83(0.74-0.97)$ & $<0.001$ & $16.1 \%$ \\
\hline Cardiovascular & $0.34(0.26-0.38)$ & $<0.001$ & $10.2 \%$ \\
\hline Pulmonary & $0.78(0.65-0.97)$ & 0.001 & $16.5 \%$ \\
\hline Stroke & $0.12(0.07-0.18)$ & $<0.001$ & $6.1 \%$ \\
\hline Other & $0.50(0.42-0.59)$ & $<0.001$ & $13.3 \%$ \\
\hline \multicolumn{4}{|l|}{ Initial site of care } \\
\hline Home & - & - & $25.2 \%$ \\
\hline Nursing home & $0.60(0.52-0.67)$ & $<0.001$ & $18.4 \%$ \\
\hline Hospice unit & $0.15(0.13-0.18)$ & $<0.001$ & $8.5 \%$ \\
\hline Other & $1.44(1.16-1.81)$ & $<0.001$ & $32.4 \%$ \\
\hline Women & - & - & $22.2 \%$ \\
\hline Men & $1.21(1.06-1.35)$ & $<0.001$ & $26.8 \%$ \\
\hline \multicolumn{4}{|l|}{ DNR status } \\
\hline No & - & & $24.1 \%$ \\
\hline Yes & $0.68(0.56-0.82)$ & $<0.001$ & $16.3 \%$ \\
\hline \multicolumn{4}{|l|}{ Race } \\
\hline Non-white & - & - & $25.1 \%$ \\
\hline White & $2.80(2.55-3.03)$ & $<0.001$ & $30.1 \%$ \\
\hline
\end{tabular}

*Final PPS scores are adjusted for initial PPS score, length of stay (0-7 days; 8-14 days; 15-90 days; >90 days), hospice, diagnosis, initial site of care, gender, DNR status, and race. 